\title{
Reconfigurable Wideband Patch Antenna for Cognitive Radio
}

\author{
H. F. AbuTarboush ${ }^{1}$, S. Khan ${ }^{1}$, R. Nilavalan ${ }^{1}$, H. S. Al-Raweshidy ${ }^{1}$ and D. Budimir ${ }^{2}$ \\ ${ }^{1}$ Wireless Network and Communication Centre (WNCC), School of Engineering and Design, Brunel University, West London, \\ $U B 83 P H, U K$. \\ Hattan. AbuTarboush@brunel . ac.uk
}

\author{
${ }^{2}$ Wireless and communication Group, School of Electronic, Communication Engineering, Westminster University, London W1W \\ $6 U W, U K$ \\ D.Budimir@wmin.ac.uk
}

\begin{abstract}
Cognitive radio communication is envisaged to be a new paradigm of methodologies for enhancing the performance of radio communication systems through the efficient utilization of radio spectrum. A key enabler for realization of a cognitive communication system is the capability of re-configurability in the underlying hardware and the associated protocol suite. Reconfigurable double C-Slot microstrip patch antenna fed by $\mathbf{5 0}$ $\Omega$ microstrip line is proposed in this paper. The frequency tuning is performed by switching on and off two patches. The antenna can operate in dual-band or in very wide band mode in 5, 6 and 7 GHz bands. The wide-band mode can be obtained when both switches are in the ON state with impedance bandwidth of $\mathbf{3 3 . 5 2}$ $\%$ from 4.99 to $7 \mathrm{GHz}$. The total size of the ground plane is $50 \mathrm{x}$ $50 \mathrm{~mm}^{2}$. The proposed antenna verified through both numerical simulation and measurement of an experimental prototype. The antenna achieves a gain of 5 to $8 \mathrm{dBi}$ and radiation efficiency about $80 \%$.
\end{abstract}

\section{INTRODUCTION}

Cognitive radio communication is envisaged to be a new/unconventional paradigm of methodologies for enhancing the performance of radio communication systems through the efficient utilization of radio spectrum. The driving force behind the idea of cognitive communication is the motivation of efficient and intelligent utilization of the radio spectrum. Owing to a number of possible methodologies for achieving the objectives associated with cognitive radio communication, it is very difficult to restrict its definition to a particular system specification. However, there are common traits of cognitive communication systems, for instance, according to [1], a cognitive communication system is an intelligent communication system, capable of learning from its radio environment and accordingly adapting its operational parameters for reliable communication and efficient utilization of radio spectrum. In order for a communication system to be intelligent, capable of learning, adaptive, and reliable (thus cognitive) there is a need of joint cooperation between several protocols across the layers. Learning from the environment constitutes an important part of a cognitive radio communication system. The learning phase employs many (hard and soft) parameters; for instance, a cognitive radio should be capable of sensing the spectrum over a wide range of frequencies and then combining the information gathered from sensing (hard parameters), with (optionally) using various soft-parameters (e.g. user preference, protocols' interaction). The hard and soft parameters of the learning stage work as an input to the decision making module. Such a decision- making module is deemed to be intelligent so that it can take an appropriate decision according to the input parameters. The behaviour of the communication system in terms of its operational parameters has to be adaptive in order to support the decisions of a decision making module. Increasing the adaptability of the overall communication system comes at the price of higher protocol/hardware complexity; nevertheless, higher adaptability would imply the possibility of higher degrees of cognition in the system.

It is important to notice that a key enabler for realization of the learning phase, more specifically for gathering the hardparameters, is the capability of re-configurability in the underlying hardware and the associated protocol suite [2]. For accomplishing the spectrum sensing the underlying hardware (antenna) should be capable of operating over a wide range of frequencies. The decision making module may then direct the actual transmitter to operate at a particular frequency band. As the 'cognitive communication' is still in its evolutionary research phases, there is no specification for the underlying hardware which should conform to the specification of a Cognitive communication system. However, the use of wideband antennas for spectrum sensing and narrow band antennas for transmission has been proposed by the research community [3-4].

From the antenna design perspective, there is an increase in the demand for multi wide- band antennas which can be easily integrated with the communication system. Electronic reconfigurability is usually achieved by incorporating switches, variable capacitors or phase shifters in the topology of the antenna. Most frequently, lumped components such as PIN diodes, varactor diodes, or MEMS switches or varactors are used in the design of reconfigurable antennas.

There are three different categories of reconfigurable antenna; the first type being frequency reconfigurable. The aim of tuning the frequency of the antenna is to have single multifunctional antenna as a small terminal for many services. In [5] a tuning method has been introduced to tune dual band 
for mobile applications. In [6] reconfigurable dual-band antenna for wireless applications has been reported with very wide range tenability. In [7] reconfigurable patch antenna for satellite and terrestrial application has been reported. From all the above papers, the radiation patterns of these antennas remain unchanged when the frequencies are tuned from one band to another. The second type of reconfigurable antenna is radiation patterns re-configurability, where the frequency band remains unchanged while the radiation pattern changes upon the system requirements. The antenna can steer their radiation patterns beams to different direction. These types of re-configurability have been reported recently in [8-10]. The third type is polarization reconfigurable. This type can provide improvements to the signal reception performance in a multipath fading environment as well as providing an additional degree of freedom to improve link quality as a form of switched antenna diversity. A reconfigurable microstrip patch antenna with switchable circular polarization using a piezoelectric transducer (PET) is reported in [11].

This paper focuses on the frequency reconfigurable antennas. It introduces reconfigurable patch antenna with a C-slot shape, which is capable of operating in dual band or wideband operations. The antenna design as proposed herein, can operate at either of the frequency bands used in 5, 6 and 7 $\mathrm{GHz}$ with very wideband impedance bandwidth.

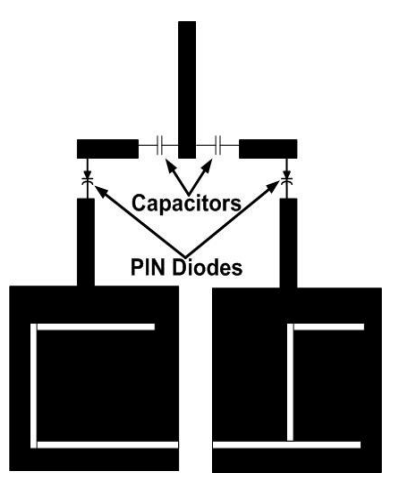

(a)

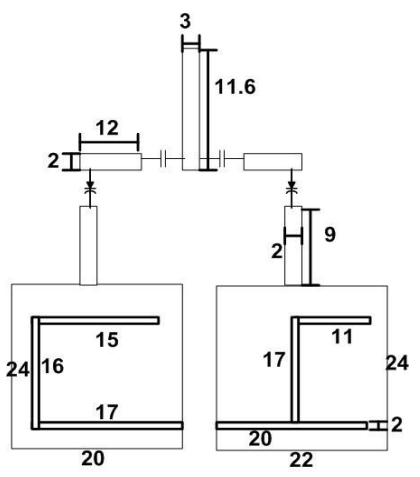

(b)
Fig. 1 Geometry of the proposed antenna. (a) Top view. (b) Dimensions.

\section{ANTENNA CONFIGURATION AND DESIGN CONCEPT}

The schematic diagram of the reconfigurable antenna is shown in fig. 1(a). It consists of two patches with the feeding configuration existing in the centre of them, two pin-diode switches and two chip capacitors. The patch antenna design was supported with a model built using a high frequency structure simulator (HFSS) based on finite elements modelling (FEM). Double C-slot shape is employed to help the operating frequencies to generate wider impedance bandwidth and to induce multiple resonance at different frequencies. The antenna is designed to operate in the 5 to $7 \mathrm{GHz}$ band. Two switches were attached to the feed of each patch. Two dc block chip capacitors with $10 \mathrm{PF}$ were placed near the $50 \mathrm{ohm}$ feeding line to block the dc connection. The dielectric material selected for the design was FR-4 which has a dielectric constant of 4.4 and height of dielectric substrate $\mathrm{h}$ is $1.57 \mathrm{~mm}$. The structure and the dimensions of the proposed antenna are shown in fig. 1(b). The proposed antenna occupies a total size of $36 \times 46 \mathrm{~mm}^{2}$. It can cover any application requiring wide impedance bandwidth in the 5 to $7 \mathrm{GHz}$ bands.

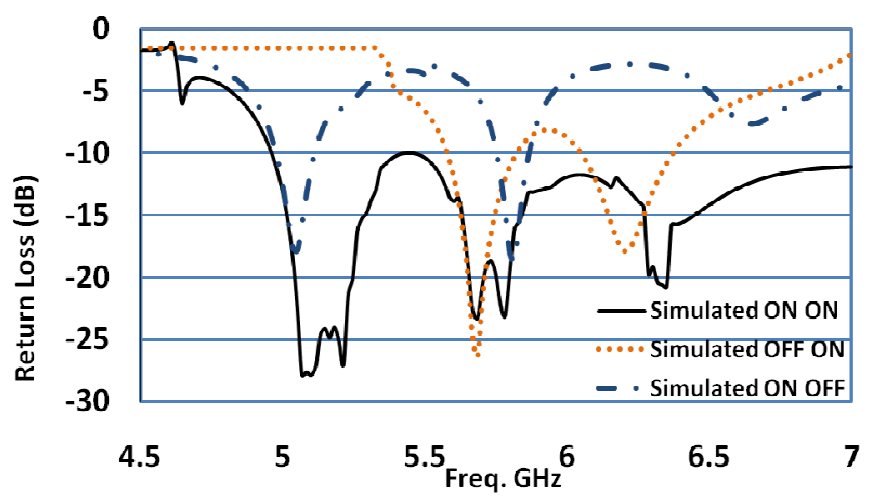

Fig. 2 Simulated return loss $S_{11}$ for different states

\section{SIMULATION AND EXPERIMENTAL RESULTS}

A. Simulation

The diode was modelled in the simulation with RLC boundary sheet. The values of the PIN diode when the switches are in the ON state is $0.9 \Omega$ and in the OFF state is $0.3 \mathrm{PF}$. These values were given in the data sheet (SMP1320-079, Skyworks Solutions Inc). The total size of the switches is $1.5 \times 0.7 \mathrm{~mm}^{2}$. Three possible states can be obtained from the two switches. When switch 1 is ON and switch 2 is OFF, dual-band can be obtained at 5 and $5.7 \mathrm{GHz}$, which can cover the Wireless Local Area Network (WLAN) application. When switch 1 is in the OFF state and switch 2 is in the ON state the dual-band is shifted toward $5.3 \mathrm{GHz}$, and $6.5 \mathrm{GHz}$. When both switches are in the ON state, the current path can travel longer, which results in a very wide bandwidth covering from $4.9 \mathrm{GHz}$ to 7 $\mathrm{GHz}$ as shown in fig. 2 and table I. The patch dimensions have direct influence on the operating frequency and on the antenna gain. The patch dimension is related to the fringing fields together with the small size of the ground plane used. The estimated bandwidths obtained from the simulation tools when both switches are in the ON state under the criterion of $\mathrm{S}_{11}$ less than $-10 \mathrm{~dB}$ is $33.52 \%$ covering from 4.99 to $7 \mathrm{GHz}$. The corresponded impedance bandwidth for the dual band when the switch is in the OFF ON state is $5.2 \%$ and $4.85 \%$ respectively, whereas in the ON OFF state the impedance bandwidth is $4.2 \%$ and $2.4 \%$ respectively as shown in table II.

TABLE I

THE IMPEDANCE BANDWIDTH FOR THE GENERATED BANDS

\begin{tabular}{|c|c|c|}
\hline $\begin{array}{c}\text { The States of } \\
\text { the Switches }\end{array}$ & $\boldsymbol{f}_{1}$ & $\boldsymbol{f}_{2}$ \\
\hline ON OFF & $5 \%$ & $25 \%$ \\
\hline OFF ON & $22 \%$ & $33 \%$ \\
\hline ON ON & \multicolumn{2}{|c|}{$33.52 \%$} \\
\hline
\end{tabular}




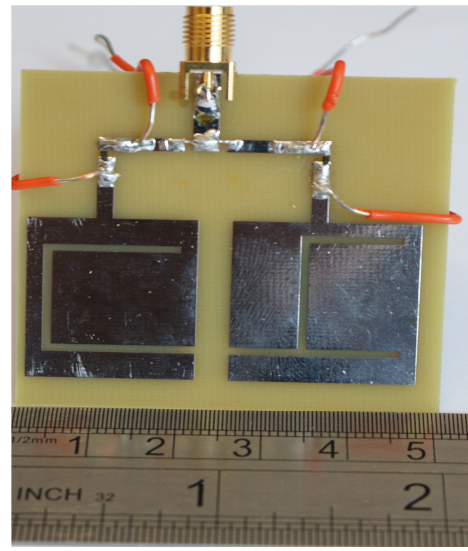

Fig. 3 A prototype of the proposed antenna

\section{B. Experimental}

In order to validate the simulation results, the proposed antenna has been fabricated according to the specifications given in the previous section. fig. 3 shows the prototype of the antenna. The proposed antenna was fabricated, tested, and compared with simulated results. The return loss was measured using Agilent N5230A vector network analyser. In fig. 4 , the simulated values of the $S_{11}$ in the final design are compared with the measured data. It was found that, the simulated and measured results are in good agreements. The discrepancy between the simulated and measured result might be attributed to the fabrication process.

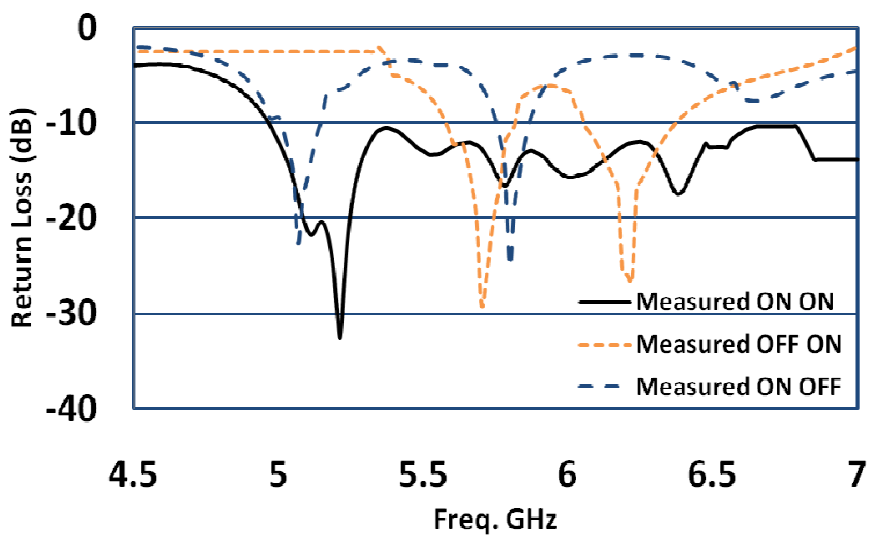

Fig. 4 Measured return loss $S_{11}$ for the proposed antenna

TABLE II

THE RESONANT FREQUENCIES GENERATED FROM EACH STATES

\begin{tabular}{|c|c|c|}
\hline $\begin{array}{c}\text { The States of } \\
\text { the Switches }\end{array}$ & $\boldsymbol{f}_{1}$ & $\boldsymbol{f}_{2}$ \\
\hline ON OFF & $5 \mathrm{GHz}$ & $5.7 \mathrm{GHz}$ \\
\hline OFF ON & $5.7 \mathrm{GHz}$ & $6.2 \mathrm{GHz}$ \\
\hline ON ON & \multicolumn{2}{|c|}{ Wideband covering from $4.99 \mathrm{GHz}$ to $7 \mathrm{GHz}$} \\
\hline
\end{tabular}

The radiation patterns is an important parameter for the antenna, therefore, the $\mathrm{E}$ and $\mathrm{H}$ plane for the three states are plotted as shown in figures 5, 6 and 7. It can be observed from the radiation patterns that there is a stable response throughout the operating bands with low cross polarization. The maximum gains for the operating frequencies in all the states are shown in fig. 8. As another key performance in antenna design, the total radiation efficiency is plotted in fig. 9. It can be seen that the total radiation efficiencies are above $80 \%$ in all the different states of the switches.
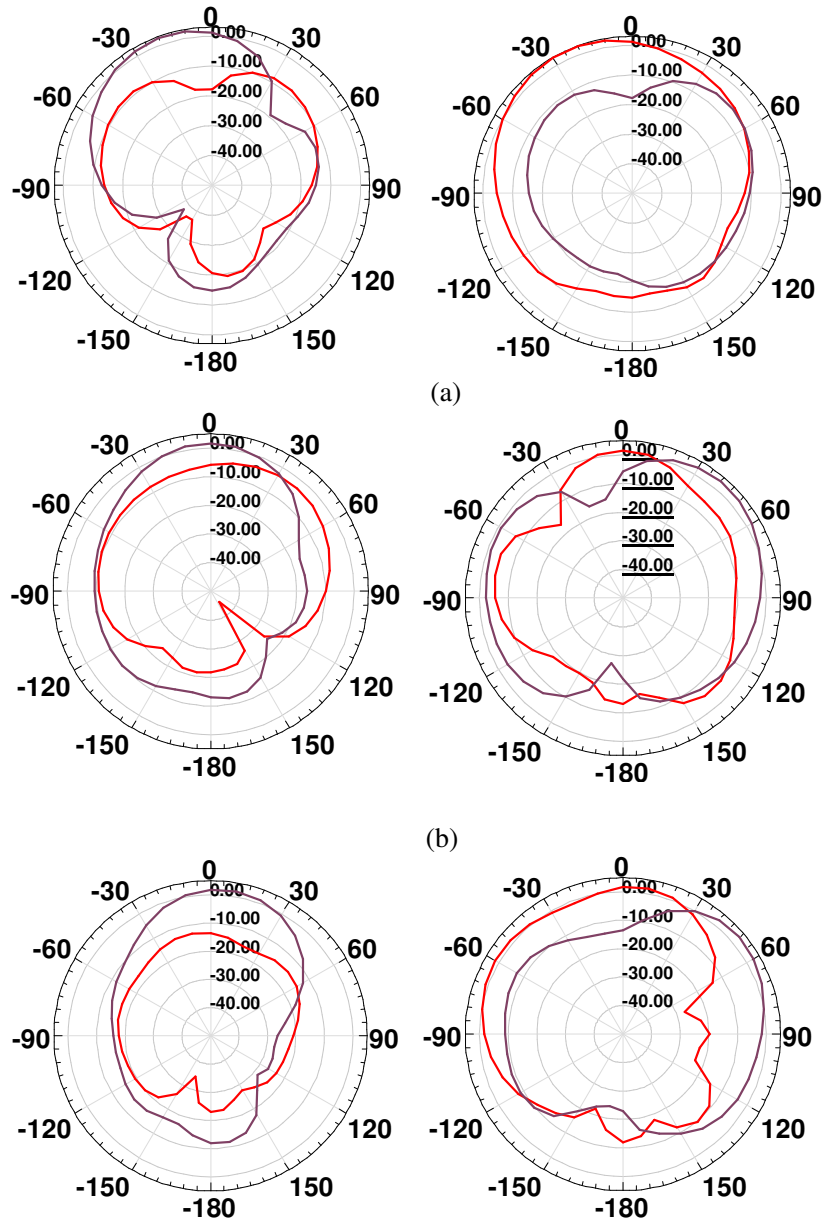

(c)

Fig. $5 \mathrm{E}$ and H Plane Radiation Patterns when the switch is in the ON ON State (a) $5.2 \mathrm{GHz}$, (b) $5.7 \mathrm{GHz}$ and (c) $6.5 \mathrm{GHz}$
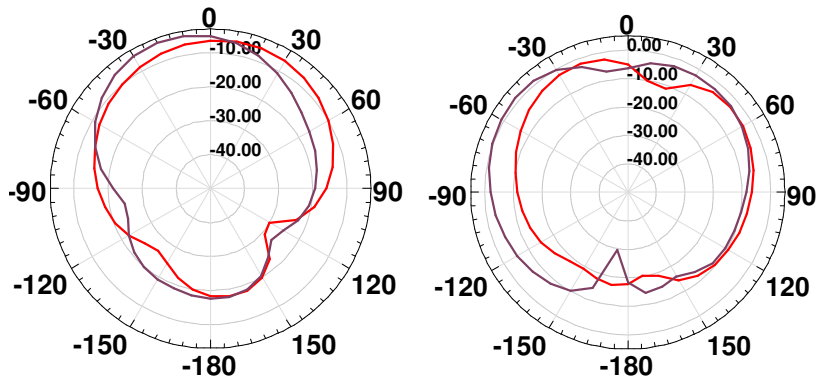

(a) 

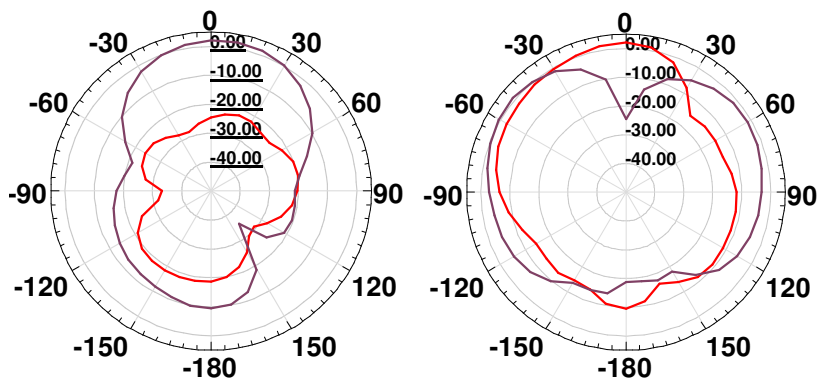

(b)

Fig. $6 \mathrm{E}$ and $\mathrm{H}$ Plane Radiation Patterns when the switch is in the OFF ON State (a) $5.6 \mathrm{GHz}$ and (b) $6.5 \mathrm{GHz}$
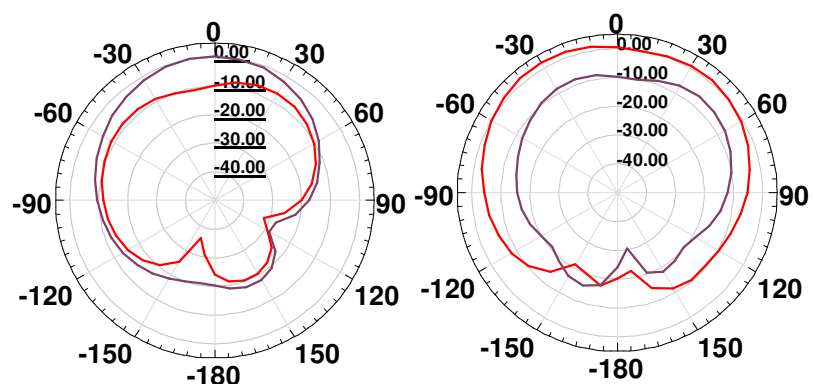

(a)
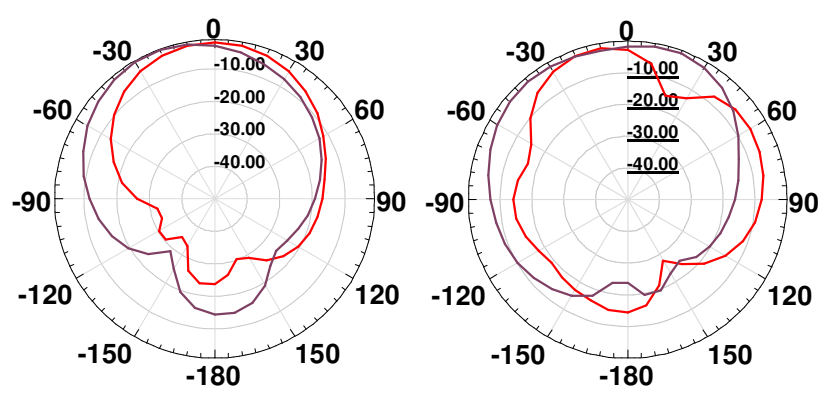

(b)

Fig. $7 \mathrm{E}$ and H Plane Radiation Patterns when the switch is in the ON OFF State (a) $5.2 \mathrm{GHz}$ and (b) $5.7 \mathrm{GHz}$

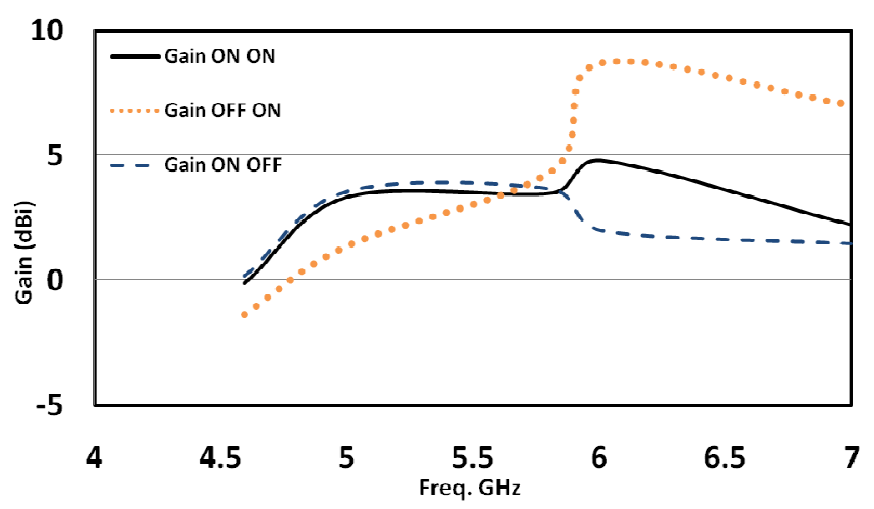

Fig.8 Maximum gain for the proposed antenna with the three switches

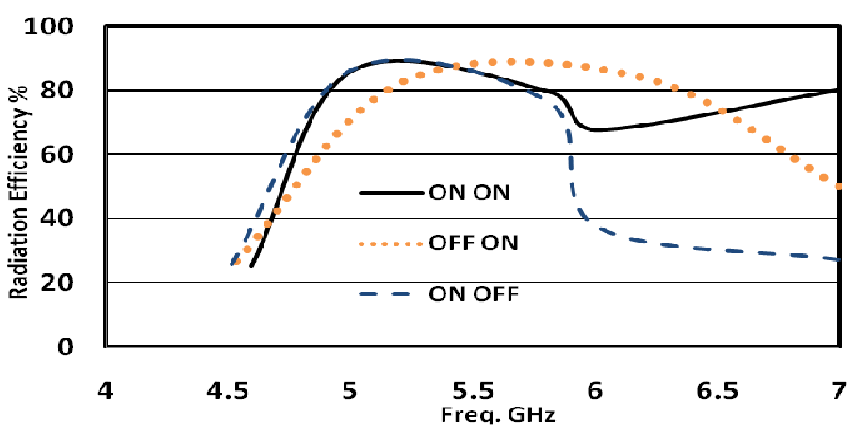

Fig. 9 Total efficiency of the proposed antenna with the three switches

\section{CONCLUSIONS}

A dual-band reconfigurable double $\mathrm{C}$-slot antenna is proposed in this paper. Two PIN diode switches are applied to the design to generate a dual-band and wide band by changing the states of the PIN diode. The radiation patterns for the three states are considered at both bands and over the entire frequency range of the antenna. Low levels of cross-polarized radiation are observed, and the radiation pattern of each band remains practically unchanged as the state of the switches is changed. The proposed antenna verified through both numerical simulation and measurement of an experimental prototype. The antenna achieves a gain of 5 to $8 \mathrm{dBi}$ and radiation efficiency of $80 \%$.

\section{REFERENCES}

[1] S. Haykin, 'Cognitive radio: brain-empowered wireless communications', Selected Areas in Communications, IEEE Journal on, Vol. 23, No. 2. (2005), pp. 201-220.

[2] M. Dillinger, N. Alonistioti, and K. Madani, Software Defined Radio: Architectures, Systems and Functions, Wiley, 2003.

[3] J. Kelly, E. Ebrahimi, P. S. Hall, P. Gardner, and F Ghanem, "Combined wideband and narrowband antennas for cognitive radio applications," in The IET seminar on Cognitive Radio and Software Defined Radios: Technologies and techniques, London, UK, Sep. 2008.

[4] P. S. Hall, P. Gardner, J. Kelly, E. Ebrahimi, M. R. Hamid, and F. Ghanem, "Antenna challenges in cognitive radio," in Proc. ISAP 08 , Taiwan, Oct. 2008.

[5] M. Komulainen, M. Berg, H. Jantunen, E. T. Salonen and C. Free, "A Frequency Tuning Method for a Planar Inverted-F Antenna," Antennas and Propagation, IEEE Transactions on, vol. 56, pp. 944-950, 2008.

[6] N. Behdad and K. Sarabandi, "Dual-band reconfigurable antenna with a very wide tunability range," Antennas and Propagation, IEEE Transactions on, vol. 54, pp. 409-416, 2006.

[7] M. Ali, A. T. M. Sayem and V. K. Kunda, "A Reconfigurable Stacked Microstrip Patch Antenna for Satellite and Terrestrial Links," Vehicular Technology, IEEE Transactions on, vol. 56, pp. 426-435, 2007.

[8] Symeon Nikolaou, R. Bairavasubramanian, C. Lugo Jr., I. Carrasquillo, D. C. Thompson, G. E. Ponchak, J. Papapolymerou and M. M. Tentzeris, "Pattern and frequency reconfigurable annular slot antenna using PIN diodes," Antennas and Propagation, IEEE Transactions on, vol. 54, pp. 439-448, 2006.

[9] Chien-Jen Wang and W. -. Tsai, "A slot antenna module for switchable radiation patterns," Antennas and Wireless Propagation Letters, IEEE, vol. 4, pp. 202-204, 2005.

[10] Shing-Hau Chen, Jeen-Sheen Row and Kin-Lu Wong, "Reconfigurable Square-Ring Patch Antenna With Pattern Diversity," Antennas and Propagation, IEEE Transactions on, vol. 55, pp. 472-475, 2007.

[11] S. -. Hsu and Kai Chang, "A Novel Reconfigurable Microstrip Antenna With Switchable Circular Polarization," Antennas and Wireless Propagation Letters, IEEE, vol. 6, pp. 160-162, 2007. 